\title{
Effects of Ferrite Growth Rate on Interphase Boundary Precipitation in V Microalloyed Steels
}

\author{
Toshio MURAKAMI, ${ }^{1,3) *}$ Hitoshi HATANO, ${ }^{1,3)}$ Goro MIYAMOTO ${ }^{2,3)}$ and Tadashi FURUHARA ${ }^{2,3)}$ \\ 1) Kobe Steel LTD., Kobe, Hyogo,651-2271 Japan. E-mail: murakami.toshio1@kobelco.com, hatano.hitoshi@kobelco.com \\ 2) Tohoku University, Sendai, Miyagi,980-8577 Japan. E-mail: miyamoto@imr.tohoku.ac.jp, furuhara@imr.tohoku.ac.jp \\ 3) Consortium of the Japan Research and Development Center for Metals for Fundamental Studies on Technologies for Steel \\ Materials with Enhanced Strength and Functions, Tokyo, 105-0003 Japan.
}

(Received on September 29, 2011; accepted on January 5, 2012)

\begin{abstract}
The interphase boundary precipitation behavior of vanadium carbide during isothermal ferrite transformation, which is the important phenomena for the hot forged medium carbon steels, was investigated and modeled. It was found that the intersheet spacing of interphase boundary precipitation of VC decreased with a decrease of ferrite growth rate during isothermal transformation. As a major factor affecting such an interphase boundary precipitation behavior, it was deduced that the vanadium segregation on migrating austenite/ferrite interphse boundary is quite important to understand the repeated precipitation of VC. In numerical simulation of VC precipitation, the influence of the vanadium concentration on the precipitation behavior at austenite/ferrite interface during isothermal transformation was examined based on a time-dependent solute drag model incorporated with parabolic growth rate. It was found that the change of the intersheet spacing during ferrite growth can be simulated by the newly proposed model for interphase boundary precipitation of alloy carbide.
\end{abstract}

KEY WORDS: interphase boundary precipitation; vanadium carbide; medium carbon steel.

\section{Introduction}

Use of interphase boundary precipitation of alloy carbide is known to be effective for strengthening steels. In this phenomenon, nano-sized alloy carbides can be dispersed on sheets regularly spaced in ferrite densely, so high precipitation hardening can be obtained. Therefore interphase boundary precipitation is commonly used in various steels products including microalloyed steels. In order to control precipitation hardening by interphase boundary precipitates, it is needed to reveal the mechanism of and to propose the prediction model for interphase boundary precipitation.

It was shown that the intersheet spacing of carbide precipitation was affected by the continuous cooling rate, ${ }^{1,2)}$ the isothermal transformation temperature ${ }^{3-5)}$ and the chemical composition. ${ }^{3,5)}$ According to these studies, the intersheet spacing of interphase boundary precipitation depends on the diffusion of the substitutional element that constitutes alloy carbide or the driving force for nucleation of carbide.

In order to explain such effects on the intersheet spacing, some quantitative models were proposed. ${ }^{6-13)}$ In the model proposed by Todd et al., ${ }^{6-8)}$ the intersheet spacing of interphase boundary precipitation is determined by the diffusion profile of the solute concentration in the front of the growing interphase boundary of a "pseudophase", as which they defined the sheet region. Contrarily Rios ${ }^{9)}$ considered the intersheet spacing in vanadium steels is decided by the balance between vanadium flux entering into "vanadium diffusion zone" through the austenite/ferrite interface at the front of ledge and vanadium amount included in interphase precipitates which was formed at the neighbor of vanadium diffusion zone. Lagneborg ${ }^{10)}$ and Zajac ${ }^{11)}$ suggested that vanadium was partitioned at the front of growth ledge like discontinuous precipitation, and the distance in which vanadium could diffuse during advance of growth ledge should be the ledge height, in other words the intersheet spacing. $\mathrm{Liu}^{12)}$ considered the array of interphase boundary precipitation was formed by repetition of pinning by VC precipitates nucleated at the austenite/ferrite interface and unpinning by the increase of ferrite transformation driving force which was raised by the reduction of the carbon concentration in austenite because of VC growth at the interface. These models have successfully reproduced temperature dependence of intersheet spacing by using some fitting parameters.

On the other hand, with regard to the influence of the ferrite growth rate, Lagneborg ${ }^{10)}$ and Okamoto ${ }^{13)}$ predicted that the intersheet spacing increases with the decrease of the interface velocity. But it is difficult to discuss the validity of this model because there were a few investigations about influence of ferrite growth rate on interphase boundary precipitation. Gray ${ }^{1)}$ reported the intersheet spacing decreased with advance of austenite/ferrite interface during continuous cooling. But it should contain both effects of the ferrite growth rate and transformation temperature. So the dependence of austenite/ferrite interface velocity on the intersheet spacing has not been cleared yet.

Therefore the purpose of this study is to examine the change of intersheet spacing of interphase boundary precip- 
itation of carbide and to propose a prediction model for intersheet spacing change during isothermal ferrite transformation.

\section{Experimental Procedure}

Table 1 shows the chemical composition of the medium carbon steel containing vanadium used in the present study. Specimens were machined to $8 \mathrm{~mm}$ diameter and $12 \mathrm{~mm}$ height and heat-treated by the thermo-mechanical treatment simulator. At first, they were heated to $1523 \mathrm{~K}$ for $600 \mathrm{~s}$ in order to dissolve all vanadium carbo-nitride and to obtain coarse grained austenite structure before ferrite transformation. It is because that large austenite grain size with planar austenite grain boundaries is suitable identification of ferrite growth direction and for avoiding effect of soft impingement on ferrite growth. The prior austenite grain size was about $400 \mu \mathrm{m}$ after the above austenization treatment. These specimens were immediately cooled to holding temperature $(923 \mathrm{~K}, 948 \mathrm{~K}, 973 \mathrm{~K})$ and kept for various periods between $1 \mathrm{~s}$ and $1800 \mathrm{~s}$ and water-quenched to freeze ferrite structure.

The specimens were etched by $3 \%$ nital so as to observe the microstructure by optical microscopy. Electron backscatters diffraction analysis of ferrite and martensite were made by using FE-SEM in order to investigate the orientation relationship between ferrite and prior austenite. VC precipitations distribution was observed by TEM, and thin foils for TEM observation were prepared by a twin-jet method.

Table 1. Chemical composition of the specimen (mass $\%$ ).

\begin{tabular}{cccccccc}
\hline $\mathrm{C}$ & $\mathrm{Si}$ & $\mathrm{Mn}$ & $\mathrm{P}$ & $\mathrm{S}$ & $\mathrm{V}$ & $\mathrm{Al}$ & $\mathrm{N}$ \\
\hline 0.43 & 0.25 & 0.75 & 0.015 & 0.014 & 0.29 & 0.034 & 0.0036
\end{tabular}

\section{Result}

Optical micrographs of the specimens held at $973 \mathrm{~K}$, $948 \mathrm{~K}$ and $923 \mathrm{~K}$ for $1800 \mathrm{~s}$ are indicated in Fig. 1. Film like grain boundary ferrites were formed along the prior austenite grain boundaries. Figures 2 and $\mathbf{3}$ shows the TEM micrographs of the interphase boundary precipitation of vanadium carbides (VC) in these grain boundary ferrites formed at various temperatures and measured sheet spacing of $\mathrm{VC}$, respectively. The particle size and the intersheet spacing of interphase boundary precipitations decrease with decline of holding temperature as reported in the literatures. $^{3,10)}$

The detailed investigation was carried out in the specimen transformed at $948 \mathrm{~K}$. Grain boundary ferrites are nucleated at prior austenite grain boundary and thickened during holding at $948 \mathrm{~K}$, and ferrite growth is interrupted by the pearlite transformation before $120 \mathrm{~s}$ (Fig. 4). Intragranular ferrite and pearlite were also observed even in the early stage of transformation $(60 \mathrm{~s})$, but it is supposed that they do not affect growth of grain boundary ferrites except $120 \mathrm{~s}$ since they are usually formed far enough from grain boundary ferrites.

Figure 5 shows a SIM image of grain boundary ferrite and corresponding (001) pole figures of the grain boundary ferrite and martensites transformed from austenite grains located on the left side ( $\gamma 1$, Fig. 5(b)) and the right side $(\gamma 2$, Fig. 5(c)) of the ferrite in the sample transformed for $10 \mathrm{~s}$. In Figs. 5(b) and 5(c), triangles indicate (001) pole figure of the grain boundary ferrite and small dots indicate (001) pole figures of martensites. (001) pole figures of martensites exhibit characteristic patterns for transformation products holding near $\mathrm{K}-\mathrm{S}$ orientation relationship with the parent phase. ${ }^{14)}$ So if (001) pole figure of the grain boundary ferrite coincide with those of martensites, it is clear that the grain


Fig. 1. Optical micrographs of specimens held at (a) $973 \mathrm{~K}$, (b) $948 \mathrm{~K}$ and (c) $923 \mathrm{~K}$ for $1800 \mathrm{~s}$.
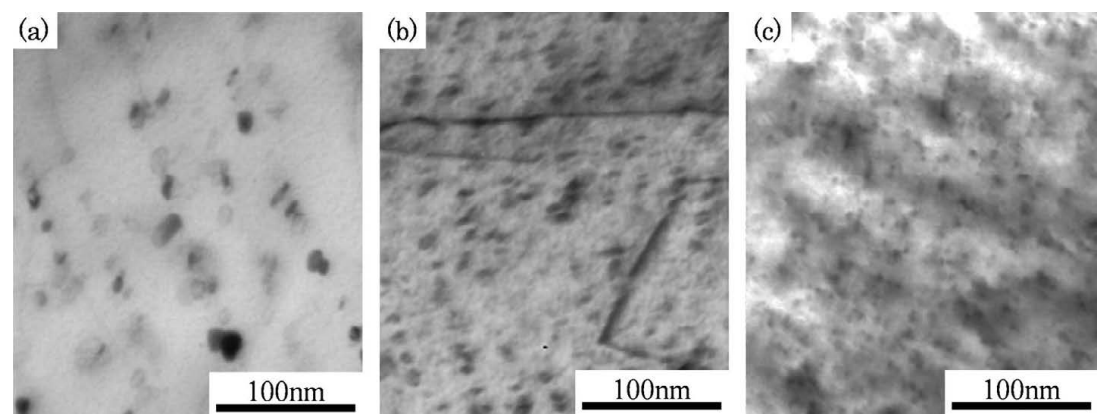

Fig. 2. TEM micrographs of specimens held at (a) $973 \mathrm{~K}$, (b) $948 \mathrm{~K}$ and (c) $923 \mathrm{~K}$ for $1800 \mathrm{~s}$. 


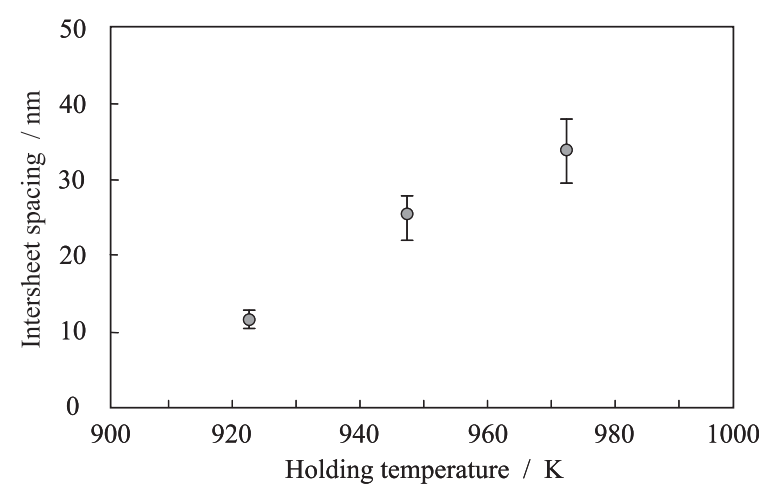

Fig. 3. Variation of intersheet spacing of interphase boundary precipitation transformed at from $923 \mathrm{~K}$ to $973 \mathrm{~K}$.
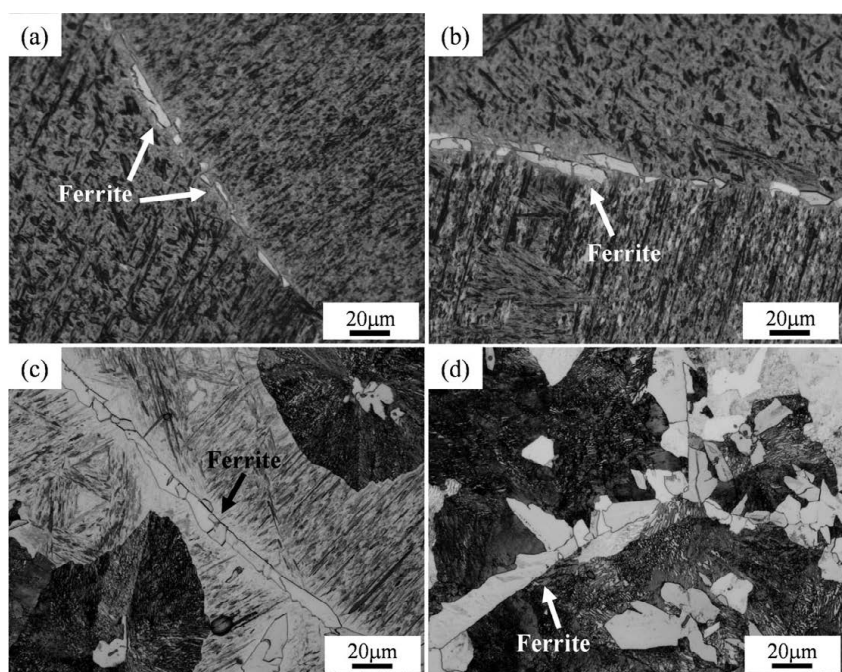

Fig. 4. Optical micrographs of specimens held at $948 \mathrm{~K}$ for (a) $10 \mathrm{~s}$, (b) $30 \mathrm{~s}$, (c) $60 \mathrm{~s}$, (d) $120 \mathrm{~s}$ boundary ferrite holds nearly $\mathrm{K}-\mathrm{S}$ orientation relationship with the prior austenite. However, the misorientation angle between martensites formed in both $\gamma 1$ and $\gamma 2$ and the grain boundary ferrite is about 10 degrees. This result means that the ferrite in Fig. 5 holds no orientation relationship with the austenites on the both side in this SIM image, however it is considered that this ferrite holds $\mathrm{K}-\mathrm{S}$ orientation relationship with austenites which exist on back side or front side of the observation plane. It has been reported that grain boundary ferrites preferentially grow into the austenite with which there is not a specific orientation relationship in carbon steels ${ }^{15)}$ and $\mathrm{V}$ added medium carbon steels. ${ }^{16)}$ Therefore it is considered that the ferrite shown in Fig. 5 grow into the both of the adjacent austenite grains.

TEM micrograph of Fig. 6 shows VC precipitation in a grain boundary ferrite having planar prior austenite/ferrite interfaces in the specimen held at $948 \mathrm{~K}$ for (a) $10 \mathrm{~s}$ and (b) $30 \mathrm{~s}$. VC are aligned parallel to the prior austenite/ferrite interface and such sheet are formed repeatedly. And it looks like that the intersheet spacing of interphase spacing is not constant in one ferrite and becomes narrower as the holding time becomes longer.

The local intersheet spacing measured Fig. 6 as a function of distance from the center of the ferrite is indicated in Fig. 7. The intersheet spacing of interphase boundary precipitations in the ferrite shows a relatively large scatter. It tends to become smaller with getting away from the center of the ferrite in both specimens transformed for $10 \mathrm{~s}$ and $30 \mathrm{~s}$. These results were different from the investigation of Okamoto et al. ${ }^{17)}$ in low carbon steels with $\mathrm{Nb}$ addition, in which the row spacing of interphase boundary precipitation was almost constant against the distance from a certain row.

There are few reports about the intersheet spacing change against the distance from the austenite/ferrite interface, so this mechanism is not clarified. But it is supposed that the

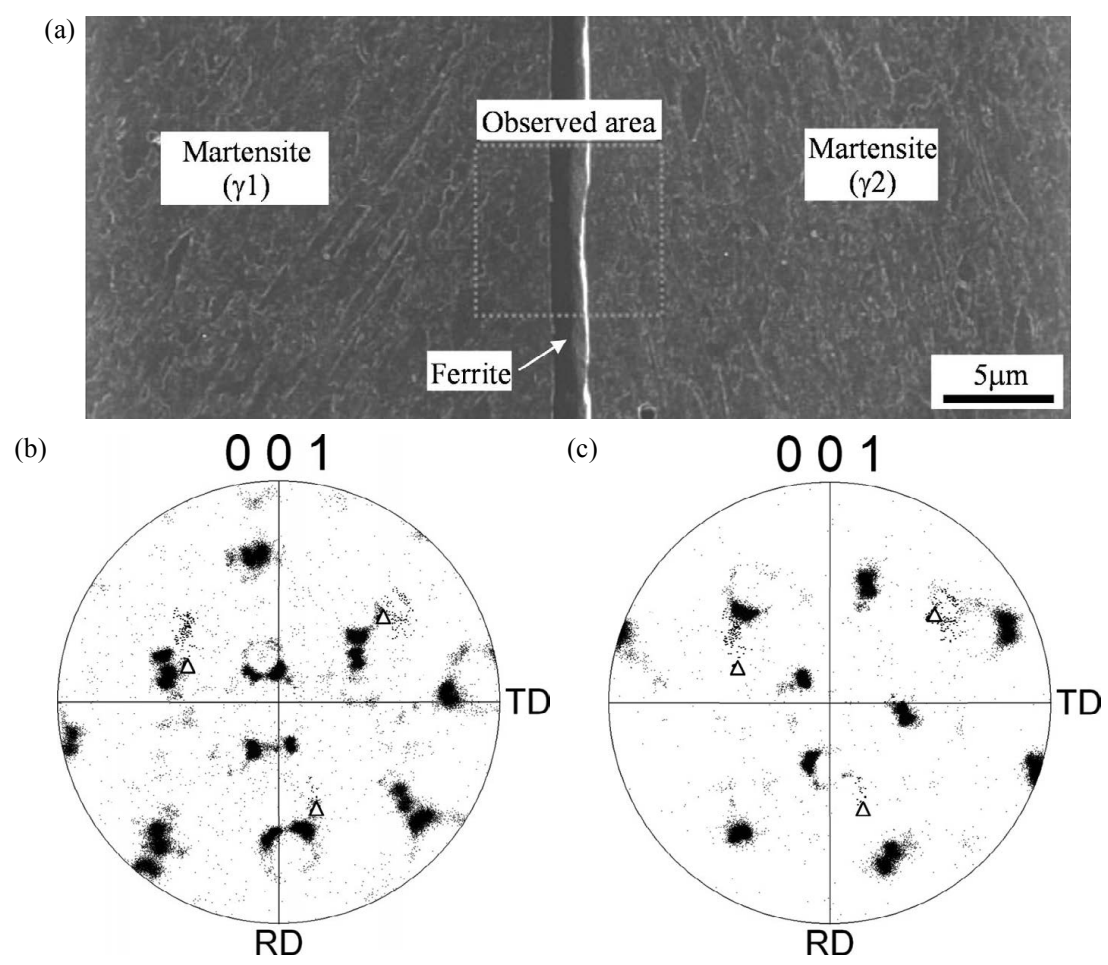

Fig. 5. (a) SIM image of the ferrite which was observed by TEM in the specimen held at $948 \mathrm{~K}$ for $10 \mathrm{~s}$, and $\{001\}$ pole figure of ferrite and martensite taken from (c) the left side and (d) the right side. 

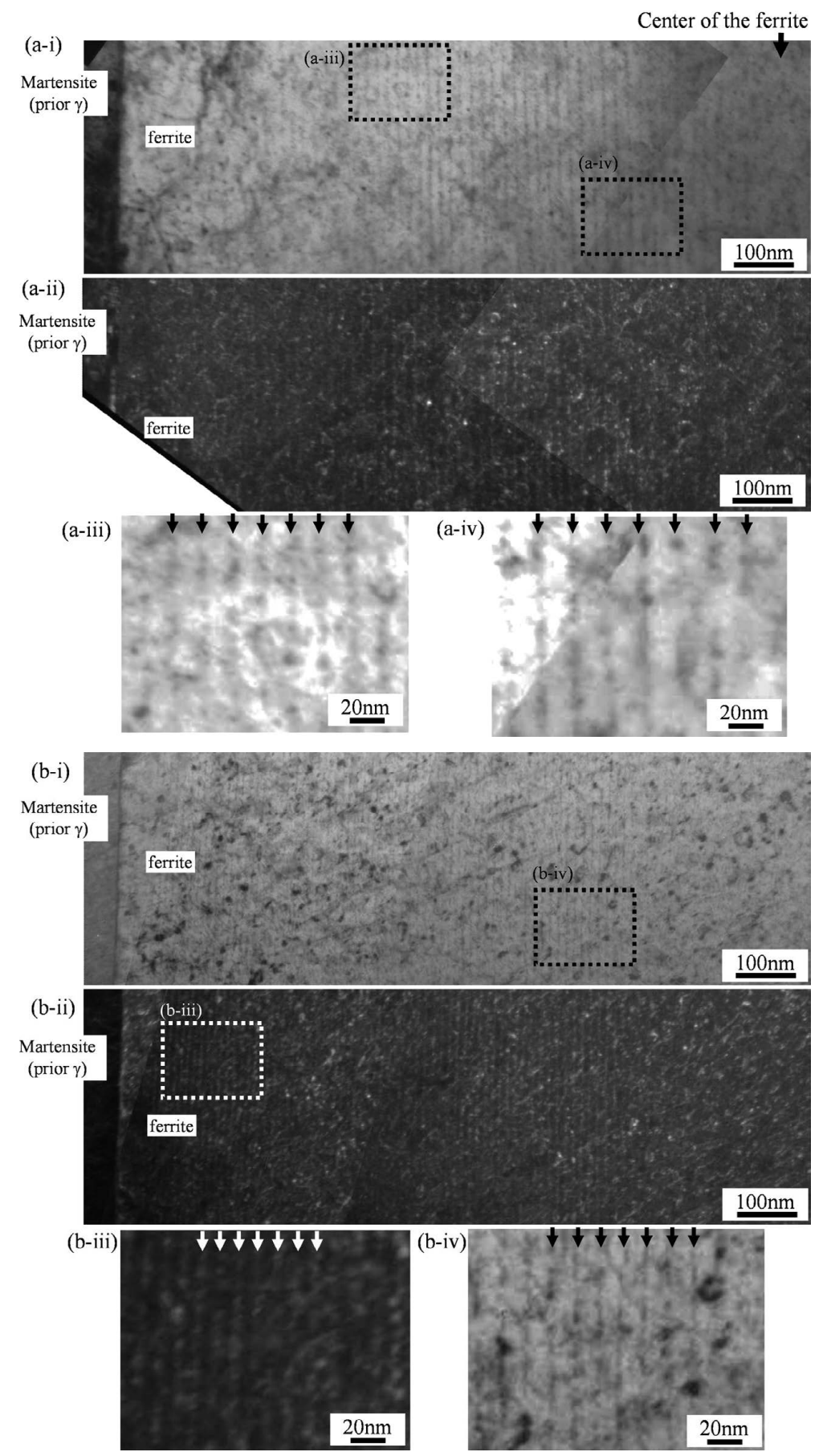

Fig. 6. TEM micrographs of (-i) bright field image and (-ii) dark field image of the $\mathrm{VC}$ in the specimens held at $948 \mathrm{~K}$ for (a) $10 \mathrm{~s}$ and (b) $30 \mathrm{~s}$, and the magnified images of the area encircled by dotted line (-iii) near and (-iv) far from the boundary in (a-i) or (b-i).

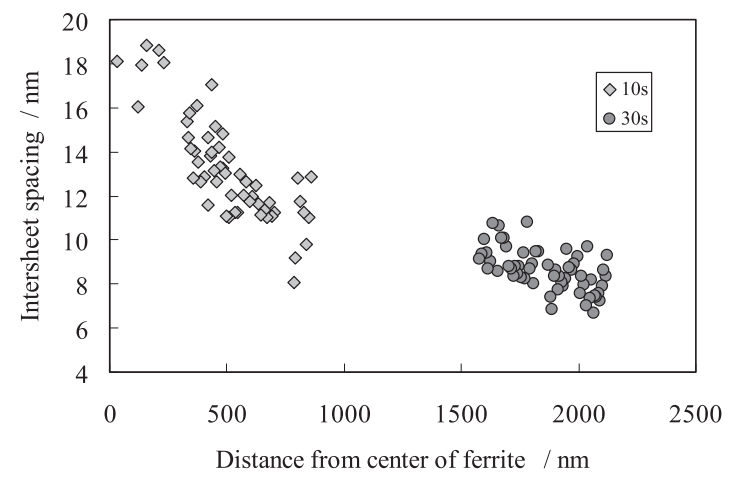

Fig. 7. Variation of intersheet spacing of interphase boundary precipitation of VC in ferrite in the specimen held at $948 \mathrm{~K}$ for $10 \mathrm{~s}$ and $30 \mathrm{~s}$. growth rate of ferrite should influence significantly on the interphase boundary precipitation, because the vanadium carbide nucleates at the interphase boundary. Usually ferrite growth is controlled by volume diffusion of carbon. The thickness of ferrite can be expressed in the linear compositional gradient model proposed by Zener as follows. ${ }^{18)}$

$$
r=\sqrt{\frac{\left(C_{\gamma}-C_{0}\right)^{2}}{\left(C_{\gamma}-C_{\alpha}\right)\left(C_{0}-C_{\alpha}\right)} D_{c}^{\gamma}} \cdot t^{0.5}
$$

Where $r$ is the half thickness of ferrite, $C_{0}$ is the initial carbon content, $C_{\gamma}$ is the para-equilibrium carbon content in austenite at the interphase boundary, $C_{\alpha}$ is the para-equilibrium carbon content in ferrite at the interphase boundary, $D_{\mathrm{c}}^{\gamma}$ is the 
diffusion coefficient of carbon in austenite and $t$ is time. The experimental result of the dependence of ferrite size against the holding time at $948 \mathrm{~K}$, which was evaluated by half thickness of grain boundary ferrites measured from observations on two orthogonal surfaces, is shown in Fig. 8. The measured ferrite thickness was in proportion to the calculated one from Eq. (1) but somewhat lower than them. Therefore it is considered that the ferrite growth rate of the $\mathrm{V}$ added steel used in this investigation can be estimated by Eq. (1).

The growth rate of ferrite $d r / d t$ can be described as a function of half thickness of ferrite.

$$
\frac{d r}{d t}=\frac{1}{2} \cdot \frac{\left(C_{\gamma}-C_{0}\right)^{2}}{\left(C_{\gamma}-C_{\alpha}\right)\left(C_{0}-C_{\alpha}\right)} D_{\mathrm{c}}^{\gamma} \cdot \frac{1}{r}
$$

By assuming that the position of the austenite grain boundary at which the ferrite was nucleated was the point of the largest intersheet spacing, the relationship between the ferrite growth rate calculated with Eq. (2) and the intersheet spacing is shown in Fig. 9. The intersheet spacing shows strong correlation to the ferrite growth rate.

According to Eq. (2), decrease in ferrite growth rate with ferrite thickness in the medium carbon steel is more significant than the low carbon steels used in the investigation conducted by Okamoto et al. ${ }^{17)}$ Therefore it is supposed that the reason why the difference of the intersheet spacing between their report and this investigation is the difference of the carbon content.

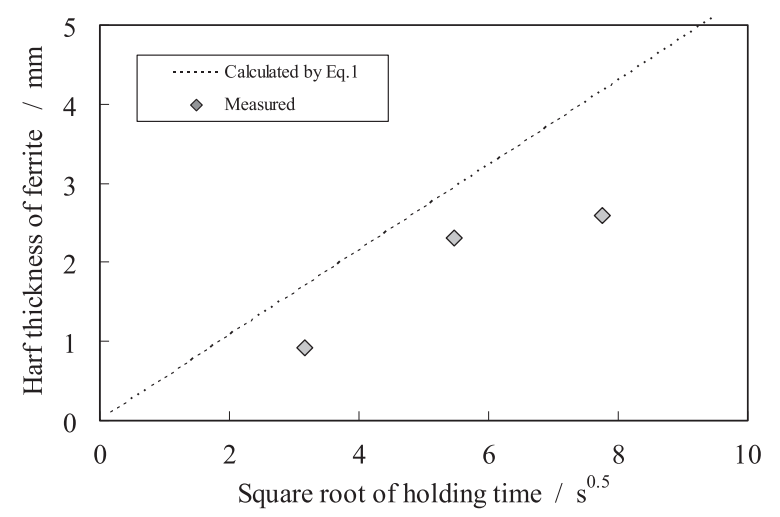

Fig. 8. Relationship between ferrite thickness and holding time at $948 \mathrm{~K}$.

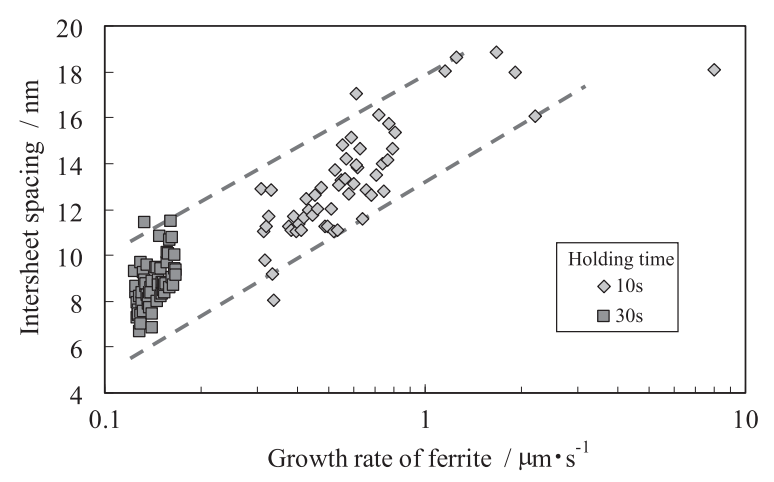

Fig. 9. Change of the intersheet spacing against the growth rate in ferrite isothermally transformed ferrite at $948 \mathrm{~K}$.

\section{Discussion}

\subsection{Validity of the Existing Model}

Todd et al. ${ }^{6-8)}$ proposed the prediction model based on the solute-drag nucleation model. In their model, the intersheet spacing is determined by the width of diffusion layer of $\mathrm{V}$. On the other hand, the model proposed by Rios ${ }^{9}$ is based on the ledge mechanism. In his model, the ledge height is determined by volume diffusion distance of $\mathrm{V}$. But both model do not consider the effect of velocity of austenite/ferrite interface and can not explain growth rate dependent intersheet spacing observed in the present study.

On the contrary, in the model provided by Liu, ${ }^{12)}$ the interphase boundary precipitation is assumed to be formed by the repetition of pinning of interface with alloy carbides nucleated due to the carbon enrichment at interface accompanied with advance of the interphase boundary, and unpinning of interface caused by increasing of ferrite transformation driving force because of the carbon consumption after carbide precipitation. And the change of the driving force is defined by the difference between chemical potential of carbon in austenite and that in ferrite at the boundary. According to this model, the intersheet spacing becomes small as the growth rate decreases, which accords with the experimental result in this study.

As stated above, it is assumed that the carbon chemical potential in the austenite and ferrite nearby the interphase boundary can be variable in his model. However it is reasonable to suppose that the local equilibrium condition should be kept at the interface during ferrite transformation. The calculated values of carbon content under olthoequlibrium are listed in Table 2. Existence of VC does not influence largely to the carbon content in austenite neighbor to the interface. This indicated that a large change of the driving force in order to make the austenite/ferrite interface un-pinned cannot be expected between the cases before and after the carbide precipitation.

On the other hand, some models based on the ledge mechanism or the quasi-ledge mechanism are proposed. In the model proposed by Lagneborg, ${ }^{10)}$ Zajac $^{11)}$ and Okamoto, ${ }^{13)}$ the intersheet spacing of carbide depends on the ledge height of the austenite/ferrite interface. And the ledge height is determined by the boundary diffusion distance of vanadium on the riser face of the growth ledge. Then the intersheet spacing $\lambda$ can be described as follows.

$$
\lambda=\left(\frac{a D_{M}^{B} l}{2 \mathrm{~K}_{1}}\right)^{1 / 2}
$$

Where $a$ is size of unit cell, $D_{M}^{B}$ is the interphase boundary diffusion coefficient of component $\mathrm{M}$ which controls the formation of interphase boundary precipitates, $l$ is distance from ferrite nucleation site, $K_{1}$ is proportional factor of diffusion-controlled ferrite growth similar to parabolic growth

Table 2. Local equilibrium carbon content at austenite/ferrite interface (mass $\%)$.

\begin{tabular}{ccc}
\hline & $\mathrm{C}(\mathrm{bcc})$ & $\mathrm{C}(\mathrm{fcc})$ \\
\hline No VC & 0.025 & 1.48 \\
VC exist & 0.025 & 1.39 \\
\hline
\end{tabular}


rate constant. According to this equation, the intersheet spacing increases as ferrite is thickened. Therefore it can be concluded that their models do not reproduce effects of ferrite growth rate on intersheet spacing observed in this work.

According to ledge mechanism or quasi-ledge mechanism, it is supposed that the intersheet spacing was controlled by ledge height. Although the minimum height of growth ledge is equal to the interplanar spacing of parallel close packed planes (ca.0.2 nm), the intersheet spacing is two orders of magnitude larger than it. It can be supposed that several ledges coalesce to form a super-ledge by some mechanisms whose height determines intersheet spacing. The theory of superledge formation was proposed by Bhadesia. ${ }^{19)}$ In his theory, superledge is formed by ferrite nucleation on an edge of ferrite allotriomorph and the height of superledge is determined by the critical nucleus height, which is derived by a function of interface energy between ferrite and austenite and the driving force for transformation. However, it is mentioned that both of the interface energy and the driving force are not changed during ferrite transformation in his paper. Therefore, the intersheet spacing should not change during isothermal transformation.

Thus the intersheet spacing change indicated in Fig. 9 can not be explained by existing models for interphase boundary precipitation. Therefore it is needed to propose new model in order to explain the behavior of interphase boundary precipitation during isothermal transformation.

\subsection{Numerical Model for Interphase Boundary Precip- itation}

Considering the change of the intersheet spacing during ferrite transformation indicated in Fig. 9, it can be said that nucleation of alloy carbide at austenite/ferrite interface is promoted by decreasing ferrite growth rate. According to the classical nucleation theory, nucleation rate $J^{*}$ can be showed in Eq. (4).

$$
\begin{aligned}
& J^{*}=N_{\mathrm{v}} \beta^{*} Z \exp \left(-\frac{\Delta G^{*}}{k T}\right) \\
& \beta^{*}=\frac{16 \pi \sigma_{\mathrm{V} C-\text { Matrix }}{ }^{2} D_{\mathrm{M}}}{a_{V C}{ }^{4} \Delta G_{\mathrm{V}}{ }^{2}} \\
& Z=\frac{V_{\mathrm{a}} \Delta G_{\mathrm{V}}{ }^{2}}{8 \pi\left(k T \sigma_{\mathrm{V} C \text {-Matrix }}{ }^{3}\right)^{1 / 2}} \\
& \Delta G^{*}=\frac{4 \sigma_{\mathrm{V} C-\text { Matrix }}-\sigma_{\alpha-\gamma}}{2 \Delta G_{\mathrm{V}}}
\end{aligned}
$$

Where $N_{\mathrm{v}}$ is the number of potential nucleation site per unit volume, $\beta^{*}$ is frequency factor, $Z$ is Zeldovich non-equilibrium factor, $\Delta G^{*}$ is the activation energy of nucleation and $k$ is Boltzmann coefficient, $V_{\mathrm{a}}$ is the atomic volume of a substitutional atom of VC, $a_{\mathrm{VC}}$ is the lattice parameter of $\mathrm{VC}, D_{\mathrm{M}}$ is the volume diffusion coefficient of component $\mathrm{V}$ in the matrix, $\Delta G_{\mathrm{V}}$ is the driving force of nucleation and $\sigma_{\mathrm{VC}-\text { Matrix }}$ is the interfacial energy between VC and the matrix. Strictly speaking, the interfacial energy should be distinguished between VC-austenite, VC-ferrite and austenite/ferrite boundaries, respectively, ${ }^{20)}$ but in this case the interfacial energy of VC-austenite and VC-ferrite were presumed as the same parameter $\sigma_{\mathrm{VC}-\text { Matrix }}$. Using $J^{*}$, the increasing rate of number of precipitation nucleated on a austenite/ferrite interface per unit volume at time $t: d N(t)$ can be described as Eq. (5).

$$
d N(t)=J^{*} A_{\alpha-\gamma} \cdot h_{\alpha-\gamma}=f\left(A_{\alpha-\gamma}, \sigma, D_{M}, T, \Delta G_{\mathrm{V}}, t\right) \ldots .
$$

Where $A_{\alpha-\gamma}$ is the area in unit volume and $h_{\alpha-\gamma}$ is thickness of austenite/ferrite interface. In order to form the array of precipitate sheet, the increasing rate of precipitation $d N(t)$ should change at an interval and the interval time should decrease as the velocity of austenite/ferrite boundary decreases.

The temperature $T$ and the diffusion coefficient $D_{\mathrm{M}}$ is constant in this case because the change of intersheet spacing takes place during isothermal transformation. The interface energy $\sigma$ should also be constant because the orientation of austenite/ferrite interface is assumed to be constant where VC nucleates based upon the observation of the parallel sheets of $\mathrm{VC}$ dispersion.

The nucleation of $\mathrm{VC}$ at sheets indicated repeated increase in driving force of precipitation. The driving force is the function of temperature, content of vanadium and carbon. In "solute drag nucleation model" adapted by Liu, the change of carbon content is considered as the main factor for the intersheet spacing. However, as stated in the previous section, it is difficult to consider the change of carbon content ahead of the interface takes place during ferrite transformation because the diffusion velocity of carbon would be high enough to reconstruct the local equilibrium instantly.

As a reason of the change of vanadium content nearby interface, two phenomena can be considered; the vanadium concentration gradient ahead of the austenite/ferrite interface because of partitioning between austenite and ferrite in order to become equilibrium content and the vanadium segregation at the austenite/ferrite interface by solute drag.

Ferrite transformation at low temperature like $948 \mathrm{~K}$ was considered to occur under para-equilibrium or NPLE (negligible-partition local equilibrium). Under paraequilibrium, the content of substitutional elements like vanadium would not change. On the other hand under NPLE, ferrite transformation was accompanied with partitioning of the substitutional elements between austenite and ferrite at the interphase boundary. Therefore the vanadium enrichment can be expected nearby the interphase boundary.

Figure 10 is an isothermal section of $\mathrm{Fe}-\mathrm{C}-\mathrm{V}$ phase diagram at $948 \mathrm{~K}$. Only BCC and FCC were considered so as to compare the equilibrium content during ferrite transformation without VC. This diagram indicates that vanadium is slightly diluted in ferrite and enriched in austenite at $948 \mathrm{~K}$. On the other hand, it can be considered that interphase precipitates nucleate on austenite/ferrite interface or in ferrite because they hold orientation relationship with ferrite. Therefore it is difficult to consider that interphase boundary precipitation is caused by vanadium petitioning in order to become equilibrium condition.

On the other hand, it was reported that segregation at austenite/ferrite boundary can lead to enrichment solute elements several times by solute drag. ${ }^{21)}$ Such a large concentration should cause large change of precipitation driving force. Therefore we can consider the interphase boundary 


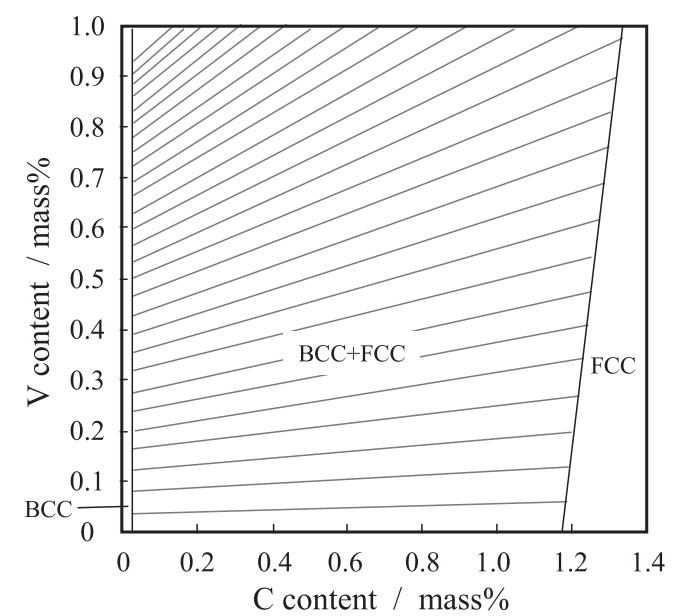

Fig. 10. Fe-rich corner of an isothermal section of $\mathrm{Fe}-\mathrm{C}-\mathrm{V}$ ternary phase diagram at $948 \mathrm{~K}$.

precipitation caused by solute drag.

In order to discuss possibility that the origin of interphase boundary precipitation is vanadium segregation at interphase boundary by solute drag, we tried to estimate vanadium segregation behavior during ferrite transformation. There are some reports about the simulation of segregation for solute drag during proeutectoid ferrite transformation. ${ }^{22-25)}$ These simulation models can consider the influence of velocity of austenite/ferrite interface and they indicate that the segregation would be promoted as the velocity decreased. The solute content reached in segregation could be several times higher than the initial content so that the precipitation at interface could be promoted strongly. Therefore, we try to develop a model in which that the segregation at the interphase boundary promote the interphase boundary precipitation.

Purdy et al. ${ }^{24)}$ proposed the simulation model for the segregation at the interphase boundary with the interaction energy between the element and the interface based on the Cahn's model ${ }^{26)}$ in order to calculate solute drag effect. According to their model, the solution $\mathrm{M}$ diffuses due to a gradient of chemical potential of solution element $\mathrm{M}$.

$$
\begin{aligned}
& F_{\mathrm{M}}=-\frac{D_{\mathrm{M}} X_{\mathrm{M}}}{k T} \frac{\partial \mu_{\mathrm{M}}}{\partial x} \\
& \mu_{\mathrm{M}}=k T \ln X_{\mathrm{M}}+E_{\mathrm{M}}
\end{aligned}
$$

Where $F_{\mathrm{M}}$ is a flux of component $\mathrm{M}, X_{\mathrm{M}}$ is the mole fraction of $\mathrm{M}, \mu_{\mathrm{M}}$ is the chemical potential, $E_{\mathrm{M}}$ is the potential energy containing the interaction energy between $M$ and the boundary, the chemical potential of pure species and the activity coefficient. Following the Fick's second law, time dependent of $\mathrm{M}$ content can be expressed as the following equation.

$$
\frac{\partial X_{\mathrm{M}}}{\partial t}=\frac{1}{k T} \frac{\partial}{\partial x}\left(D_{\mathrm{M}} X_{\mathrm{M}} \frac{\partial \mu_{\mathrm{M}}}{\partial x}\right)
$$

Equation (8) is ordinarily used to estimate the solute drag effect in a steady state. However, we solved Eq. (8) numerically at each holding time in order to examine the development of interface segregation during ferrite transformation.

In the studies by Purdy et al. ${ }^{24)}$ and Enomoto, ${ }^{21)} E_{\mathrm{M}}$ is assumed as a triangle potential well. However, this kind of
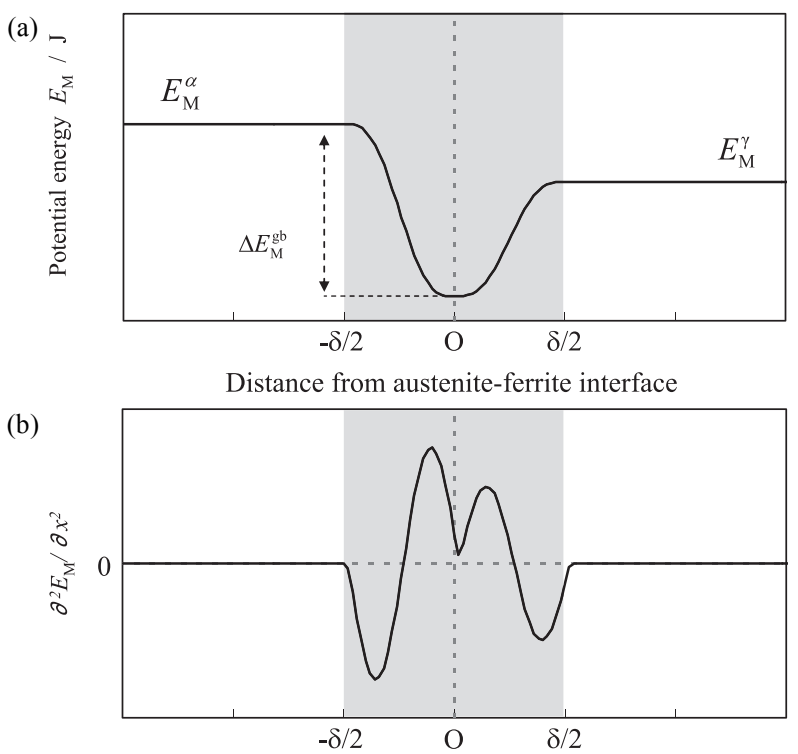

Distance from austenite-ferrite interface

Fig. 11. Schematic illustration of the energy $E_{M}$ which containing the interaction energy between solute element and austenite/ferrite interface, chemical potential of pure species and activity coefficient around austenite/ferrite interface (a) and the second derivative of $E_{\mathrm{M}}(\mathrm{x})$ with respect to the distance $\mathrm{x}(\mathrm{b})$.

potential well cannot be used for numerical calculation of Eq. (8) because this equation contains the second derivative of $E_{\mathrm{M}}$ with respect to distance which diverges to infinity at $x=0, \pm \delta / 2$. Therefore $E_{\mathrm{M}}$ is defined by Eq. (9) in order to keep continuity in the second derivative of $E_{\mathrm{M}}$, and the profile of $E_{\mathrm{M}}$ and $\partial^{2} E_{\mathrm{M}} / \partial x^{2}$ is represented in Fig. 11.

$$
\begin{gathered}
E_{\mathrm{M}}=E_{\mathrm{M}}^{\gamma} \text { at } x<x_{\mathrm{B}}-\delta / 2 \\
E_{\mathrm{M}}=E_{\mathrm{M}}^{\alpha}-\Delta E_{\mathrm{M}}^{\mathrm{B}}-\frac{2 \Delta E_{\mathrm{M}}^{\mathrm{B}} \cdot\left(x-x_{\mathrm{IB}}\right)}{\delta}-\frac{\Delta E_{\mathrm{M}}^{\mathrm{B}}}{2 \cdot \pi} \sin \left[\pi \cdot\left\{\frac{4 \cdot\left(x-x_{\mathrm{B}}\right)}{\delta}+1\right\}\right] \\
\text { at } x_{\mathrm{B}}-\delta / 2 \leq x<x_{\mathrm{B}} \\
E_{\mathrm{M}}=E_{\mathrm{M}}^{\alpha}-\Delta E_{\mathrm{M}}^{\mathrm{B}}+\frac{2\left(E_{\mathrm{M}}^{\gamma}-E_{\mathrm{M}}^{\alpha}+\Delta E_{\mathrm{M}}^{\mathrm{B}}\right) \cdot\left(x-x_{\mathrm{B}}\right)}{\delta}+\frac{\left(E_{\mathrm{M}}^{\gamma}-E_{\mathrm{M}}^{\alpha}+\Delta E_{\mathrm{M}}^{\mathrm{B}}\right)}{2 \cdot \pi} \\
\sin \left[\pi \cdot\left\{\frac{4 \cdot\left(x-x_{\mathrm{B}}\right)}{\delta}+1\right\}\right] \text { at } x_{\mathrm{B}} \leq x<x_{\mathrm{B}}+\delta / 2 \\
E_{\mathrm{M}}=E_{\mathrm{M}}^{\gamma} \text { at } x_{\mathrm{B}}+\delta / 2 \leq x \ldots \ldots \ldots \ldots \ldots . .(9)
\end{gathered}
$$

Where $E_{\mathrm{M}}^{i}=k T \ln \gamma_{\mathrm{M}}^{i}+\mu_{\mathrm{M}}^{0}(i=\alpha, \gamma), x_{\mathrm{B}}$ is the position of austenite/ferrite interface, $\delta$ is the thickness of interphase boundary, $\gamma_{\mathrm{M}}^{i}$ is the activity coefficient of $\mathrm{M}$ in phase $i, \mu_{\mathrm{M}}^{0}$ is the chemical potential of pure species $\mathrm{M}, \Delta E_{\mathrm{M}}^{\mathrm{B}}$ is the depth of potential well. The diffusion coefficient of $M$ around the interphase boundary is defined as Eq. (10) in order to keep continuity in the derivative of $D_{M}$.

$$
\begin{aligned}
& D_{\mathrm{M}}=D_{\mathrm{M}}^{\alpha} \text { at } x<x_{\mathrm{B}}-\delta / 2 \\
& D_{\mathrm{M}}=\frac{D_{\mathrm{M}}^{\mathrm{B}}-D_{\mathrm{M}}^{\alpha}}{2} \cos \left\{\frac{2 \pi\left(x-x_{\mathrm{B}}\right)}{\delta}\right\}+\frac{D_{\mathrm{M}}^{\mathrm{B}}+D_{\mathrm{M}}^{\alpha}}{2} \text { at }
\end{aligned}
$$




$$
\begin{aligned}
& x_{\mathrm{B}}-\delta / 2 \leq x<x_{\mathrm{B}} \\
& D_{\mathrm{M}}=\frac{D_{\mathrm{M}}^{\mathrm{B}}-D_{\mathrm{M}}^{\gamma}}{2} \cos \left\{\frac{2 \pi\left(x-x_{\mathrm{B}}\right)}{\delta}\right\}+\frac{D_{\mathrm{M}}^{\mathrm{B}}+D_{\mathrm{M}}^{\gamma}}{2} \text { at } \\
& x_{\mathrm{B}} \leq x<x_{\mathrm{B}}+\delta / 2 \\
& D_{\mathrm{M}}=D_{\mathrm{M}}^{\gamma} \text { at } x_{\mathrm{B}}+\delta / 2 \leq x
\end{aligned}
$$

Where $D_{\mathrm{M}}^{\alpha}, D_{\mathrm{M}}^{\gamma}, D_{\mathrm{M}}^{\mathrm{B}}$ is the diffusion coefficient of $\mathrm{M}$ in ferrite, austenite and the boundary. The position of the boundary was obtained with the growth rate of ferrite $d r / d t$ as Eq. (11).

$$
x_{\mathrm{B}}=\int_{0}^{t} \frac{d r}{d t} d t
$$

Here, the influence of $\mathrm{V}$ addition on the growth rate of ferrite is considered. It can be considered that the main factors to affect the ferrite growth behavior are pinning effect of VC precipitates and solute drag effect of solute vanadium on austenite/ferrite interface.

The pinning force $P_{\mathrm{z}}$ was estimated by the equation proposed by Gladman ${ }^{27)}$ indicated in Eq. (12).

$$
P_{\mathrm{z}}=3 f \sigma_{\text {VC-Matrix }} / 2 R
$$

Where $f$ and $R$ is the volume fraction and the radius of interphase boundary precipitates. It is known interphase boundary precipitates have $\mathrm{BN}$ orientation relationship. Therefore the interface energy can be assumed as low value about $0.1 \mathrm{~J} / \mathrm{m}^{2}$. And if it is assumed that all added vanadium precipitates as $\mathrm{VC}$ and radius of $\mathrm{VC}$ is small about $1 \mathrm{~nm}$, pinning force of interphase precipitates is about $6 \mathrm{~J} / \mathrm{mol}$. On the other hand, the driving force of ferrite transformation of this steel at $923 \mathrm{~K}$ is about $120 \mathrm{~J} / \mathrm{mol}$, much larger than estimated pinning force of interphase boundary precipitation. So it can be considered reasonable to ignore the influence of pinning effect of interphase boundary precipitation on ferrite growth behavior.

In the case of solute drag, the segregation of vanadium at austenite/ferrite interface be formed during ferrite transformation but it should disappear in the short term by the formation of interphase boundary precipitation. Therefore it is considered that the effect of solute drag on the growth rate of ferrite is not so large, too.

Actually, Capdevila ${ }^{28)}$ reported that the normal parabolic growth model under para-equilibrium is suitable for the kinetics of idiomorphic ferrite transformation in a microalloyed medium carbon steel. Therefore, in this model, the growth rate of grain boundary ferrite containing interphase boundary precipitation is calculated with the Zener's model (Eq. (1)).

Figure 12 shows the profile of vanadium concentration and Fig. 13 shows the maximum value of vanadium concentration around interphase boundary calculated with the change of the ferrite growth rate according to the Zener's model. This calculated result indicates that the V segregation at austenite/ferrite interface develops to several mol\% during migration of a few tens of nanometers. And the maximum vanadium concentration at the interphase boundary increases continuously with a decrease in boundary velocity. This vanadium enrichment is considered to promote the

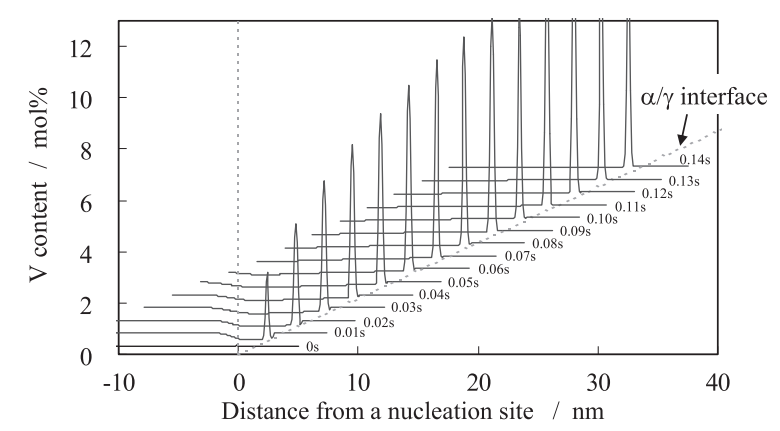

Fig. 12. Calculated result of $\mathrm{V}$ concentration profile around the austenite/ferrite interface during ferrite transformation.

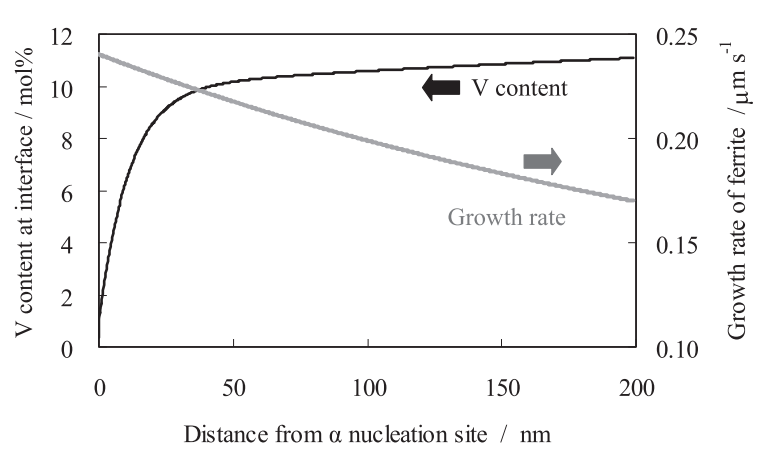

Fig. 13. V concentration at austenite/ferrite interface during ferrite transformation.

interphase boundary precipitation of VC.

Then we assume that the interphase boundary precipitation occurs when the time required for nucleation at the boundary $t^{p}$ became smaller than the time required for the interphase boundary to pass the precipitation region equal to the critical size of VC nucleation $t^{L}$. This condition can be expressed as below.

$$
\begin{gathered}
t^{L} \leq t^{p} \ldots \ldots \ldots \\
t^{L}=\frac{2 r^{*}}{d r / d t} \\
t^{p}=\frac{1}{A_{\alpha-\gamma} \cdot t_{\alpha-\gamma} \cdot J^{*}}
\end{gathered}
$$

For the calculation, there were unknown variables: interaction energy $\Delta E_{\mathrm{M}}^{\mathrm{B}}$, boundary diffusion coefficient $D_{\mathrm{M}}^{\mathrm{B}}$, density of potential nucleation site $N_{\mathrm{v}}$ and interphase boundary energy $\sigma$. Interphase boundary diffusion coefficient for vanadium has not been reported. But for $\mathrm{Cr}^{29,30)}$ the boundary diffusion coefficient is about 12000 times to diffusion coefficient in ferrite at $973 \mathrm{~K}$. Therefore we use the same ratio for the boundary diffusion of vanadium with respect to that in ferrite. Number of potential nucleation site $N v$ is taken here as the function of vanadium atoms per unit volume, i.e. the vanadium mole fraction at the boundary. Interaction energy and interface energy was used as fitting parameters in order to fit the calculation result to experimental one. Driving force of VC precipitation can not be calculated strictly because of the absence of thermodynamic database on interphase boundary. In this model, driving force of VC precipitation is derived by the thermodynamic calculation with vanadium content at interphase boundary which is calculated on the assumption that the interphase boundary pre- 


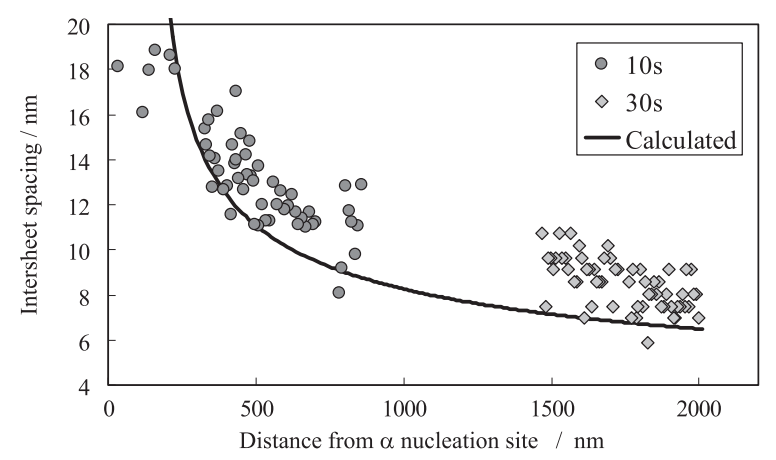

Fig. 14. Distribution of calculated intersheet spacing against the distance from the ferrite nucleation site.

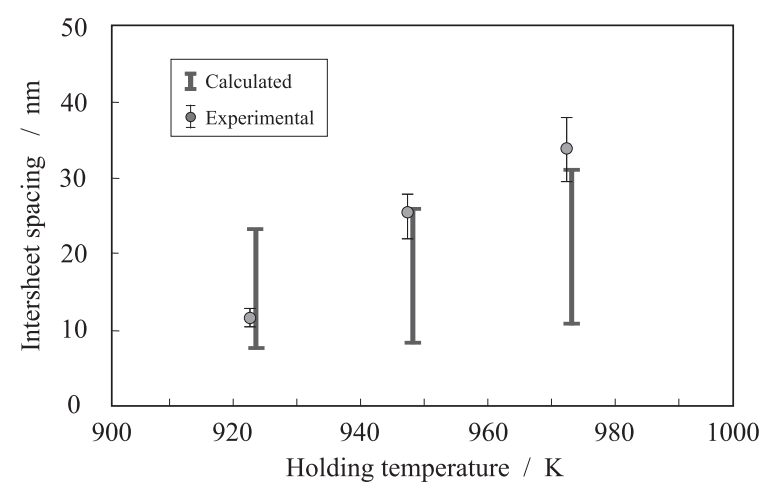

Fig. 15. Calculated intersheet spacing of interphase boundary precipitation formed between ferrite nucleation site and $1 \mathrm{~mm}$ away from it.

cipitation occurs in ferrite phase with vanadium content derived by segregation model proposed in this paper and para-equilibrium carbon content in ferrite phase, because there is good coherency between VC and ferrite matrix. And other parameters (ex. local equilibrium carbon content at austenite/ferrite interface) are derived by thermodynamic calculation. And it is assumed that the segregated vanadium on austenite/ferrite interface is consumed when interphase boundary precipitation occur.

The interphase boundary precipitation during the isothermal transformation of $0.45 \mathrm{C}-0.25 \mathrm{Si}-0.75 \mathrm{Mn}-0.3 \mathrm{~V}$ was calculated by this prediction model with fitting parameters $\Delta E_{\mathrm{M}}^{\mathrm{gb}}: 5.0 \times 10^{-20}[\mathrm{~J}], N v: 5.00 \times 10^{25}\left[\mathrm{~m}^{-3}\right]$ and $\sigma: 0.8[\mathrm{~J} / \mathrm{m} 2]$. The influence of the distance from the center of the ferrite on the intersheet spacing is indicated in Fig. 14. The calculated intersheet spacing of interphase boundary precipitation is decreased with the distance. This behavior is consistent with the experimental result in the present study. According to this calculation result, the reduction of intersheet spacing is considered to be a result of promotion of vanadium segregation at the austenite/ferrite interface due to a fall of the interface velocity accompanied with ferrite growth.

Figure 15 indicates the influence of the holding temperature on the intersheet spacing. The calculated intersheet spacing becomes smaller as the holding temperature become lower as the experimental result, but this model is not so accurate on the dependency of temperature quantitatively.

Thus, considering the segregation of vanadium on austenite/ferrite interface, the behavior that sheet spacing of interphase boundary precipitation changes not only by transformation temperature but also during isothermal transformation, which is not considered by existing models, can be predicted semi-quantitatively.

\section{Conclusion}

Interphase boundary precipitation of VC in the proeutectoid ferrite transformation in a $\mathrm{V}$ alloyed medium carbon was examined in order to clarify the dependence of the ferrite growth rate on the interphase boundary precipitation structure. Main conclusions are listed in the following.

(1) The intersheet spacing of interphase boundary precipitation became finer with decreasing the ferrite growth rate. This behavior could not be explained in the previous models proposed for interphase boundary precipitation.

(2) With the numerical simulation of time-dependent interface segregation based on Purdy's solute drag model, the concentration of vanadium and the promotion of precipitation at interface was attempted. With the assumption that the vanadium segregation at the interphase boundary was consumed by the nucleation of $\mathrm{VC}$, the change of the intersheet spacing during isothermal transformation can be predicted.

\section{Appendix}

List of symbols

a size of unit cell

$a_{V C} \quad$ lattice parameter of $\mathrm{VC}$

$A_{\alpha-\gamma} \quad$ area of austenite/ferrite interface in unit volume

$C_{0} \quad$ initial carbon content

$C_{\gamma} \quad$ para-equilibrium carbon content in austenite at the interphase boundary

$C_{\alpha}$ para-equilibrium carbon content in ferrite at the interphase boundary

$d$ distance from ferrite nucleation site,

$D_{\mathrm{c}}^{\gamma} \quad$ diffusion coefficient of carbon in austenite

$D_{\mathrm{M}} \quad$ volume diffusion coefficient of component $\mathrm{V}$

$D_{\mathrm{M}}^{\mathrm{B}} \quad$ interface boundary diffusion coefficient of component $\mathrm{M}$,

$D_{\mathrm{M}}^{\mathrm{i}} \quad$ diffusion coefficient of component $\mathrm{M}$ in phase in phase $i(i=\alpha, \gamma)$

$E_{\mathrm{M}} \quad$ potential energy of component $\mathrm{M}$ containing the interaction energy between $M$ and the boundary, the chemical potential of pure species and the activity coefficient

$E_{\mathrm{M}}^{i} \quad$ potential energy of component $\mathrm{M}$ in phase $i$ $(i=\alpha, \gamma)$

$\Delta E_{\mathrm{M}}^{\mathrm{B}} \quad$ depth of potential well

$f \quad$ volume fraction of interphase boundary precipitates

$F_{\mathrm{M}} \quad$ flux of component $\mathrm{M}$

$\Delta G^{*} \quad$ activation energy of nucleation

$\Delta G_{\mathrm{V}} \quad$ driving force of nucleation

$h_{\alpha-\gamma} \quad$ thickness of austenite/ferrite interface

$J^{*} \quad$ nucleation rate

$k \quad$ Boltzmann coefficient

$\mathrm{K}_{1} \quad$ proportional factor of diffusion-controlled ferrite growth similar to parabolic growth rate constant

$N_{\mathrm{v}} \quad$ number of potential nucleation site per unit volume 
$d N(t) \quad$ increasing rate of number of precipitation nucleated on a austenite/ferrite interface per unit volume at time $t$

$r \quad$ half thickness of ferrite

$d r / d t \quad$ growth rate of ferrite

$R \quad$ radius of interphase boundary precipitates

$t$ time

$t^{P} \quad$ time required for $\mathrm{VC}$ nucleation at the interphase boundary

$t^{L} \quad$ time required for the interphase boundary to pass the precipitation region

$V_{\mathrm{a}} \quad$ atomic volume of a substitutional atom of $\mathrm{VC}$

$x_{\mathrm{B}} \quad$ position of austenite/ferrite interface

$X_{\mathrm{M}} \quad$ mole fraction of component M

$\mathrm{Z} \quad$ Zeldovich non-equilibrium factor

$\beta^{*} \quad$ frequency factor

$\gamma_{\mathrm{M}}^{i} \quad$ activity coefficient of component $\mathrm{M}$ in phase $i$

$\delta \quad$ thickness of interphase boundary

$\lambda$ intersheet spacing of interphase boundary precipitation

$\mu_{\mathrm{M}} \quad$ chemical potential of component M

$\mu_{\mathrm{M}}^{0} \quad$ chemical potential of pure species $\mathrm{M}$

$\sigma_{\mathrm{VC}-\text { Matrix }}$ interfacial energy between $\mathrm{VC}$ and matrix

\section{Acknowledgments}

This work was carried out as a part of "Fundamental Studies on Technologies for Steel Materials with Enhanced Strength and Functions" by Consortium of the Japan Research and Development Center for Metals. Financial support from NEDO is gratefully acknowledged.

\section{REFERENCES}

1) J. M. Gray and R. B. G. Yeo: Trans. Am. Soc. Met., 61 (1968), 255.

2) G. L. DunLop, C.-J. Carlsson and G. Frimodig: Metall. Trans. A, 9A (1978), 261.

3) A. D. Batte and R. W. K. Honycombe: J. Iron Steel Inst., 211 (1973), 284.

4) N. K. Balliger and R. W. K. Honeycombe: Metall. Trans. A, 11A (1980), 421.

5) S. Zajac, T. Siwecki and M. Korchynsky: Proc. of Int. Symp. on Low Carbon Steels for the 90's, TMS, Warrendale, PA, (1993), 139.

6) J. A. Todd, P. Li and S. M. Copley: Metall. Trans. A, 19A (1988), 2133.

7) P. Li and J. A. Todd: Metall. Trans. A, 19A (1988), 2139.

8) J. A. Todd and Y.-J. Su: Metall. Trans. A, 20A (1989), 1647.

9) P. R. Rios: J. Mater. Sci., 30 (1995), 1872.

10) R. Lagneborg and S. Zajac: Metall. Mater. Trans. A, 32A (2001), 39.

11) S. Zajac: Mater. Sci. Forum, 500-501 (2005), 75.

12) W. J. Liu: Metall. Trans. A, 24A (1993), 2195.

13) T. Okamoto and J. Ågren: Acta Mater., 58 (2010), 4791.

14) S. Morito and T. Maki: Materia Jpn., 40 (2001), 629.

15) C. S. Smith: Trans. Am. Soc. Met., 45 (1953), 533.

16) G. Miyamoto, P. Behrang and T. Furuhara: Solid State Phenom., 172-174 (2011), 420.

17) T. Okamoto, A. Borgenstam and J. Ågren: Acta Mater., 58 (2010), 4783 .

18) C. Zener: J. Appl. Phys., 20 (1949), 950.

19) H. K. D. H. Bhadesia: Phys. Status Solidi (a), 69 (1982), 745.

20) M. J. Crooks and J. M. Chilton: Metall. Trans. A, 15A (1984), 1137.

21) T. Furuhara, J. Yamaguchi, N. Sugita, G. Miyamoto and T. Maki: ISIJ Int., 43 (2003), 1630.

22) M. Hillert and B. Sundman: Acta Metall., 24 (1976), 731.

23) Z.-K. Liu: Metall. Mater. Trans. A, 28A (1997), 1625.

24) G. R. Purdy and Y. J. M. Brechet: Acta Metall., 43 (1995), 3763

25) M. Enomoto: Acta Metall., 47 (1999), 3533.

26) J. W. Cahn: Acta Metall., 10 (1962), 789.

27) T. Gladman: Proc. R. Soc. London, A294 (1966), 2.

28) C. Capdevila, F. G. Caballero and C. García de Andrés: Metall. Mater. Trans. A, 32A (2001), 1591.

29) A. W. Bowen and G. M. Leak: Metall. Trans., 1 (1970), 1695.

30) A. M. Huntz, M. Aucouturier and P. Lacombe: C.R. Acad. Sci. Paris, 165 (1967), 554. 